\title{
VOF simulation studies on binary seawater droplets collision
}

\author{
Qianjian Guo ${ }^{1}$, Xiaoni $\mathrm{Qi}^{2 *}$, Qiang $\mathrm{Yin}^{2}$, Xiaohang $\mathrm{Qu}^{2}$ \\ ${ }^{1}$ College of Mechanical Engineering, Shandong University of Technology, Zibo 255049, China \\ ${ }^{2}$ College of Traffic and Vehicle Engineering, Shandong University of Technology, Zibo 255049, China
}

Corresponding Author Email: nini@alumni.sjtu.edu.cn

https://doi.org/10.18280/ijht.360348

Received: 18 December 2017

Accepted: 25 May 2018

\section{Keywords:}

adaptive mesh, droplet collision, seawater, VOF method

\begin{abstract}
Numerical simulation of the head-on collision of binary equal-sized seawater droplets was conducted by volume of fluid method for tracking the interface and an adaptive mesh for improving the calculation efficiency. In order to reveal the mechanism and the influence factors of seawater droplets in Impinging Seawater Shower Cooling Tower (ISSCT.), veracity of the numerical models was firstly validated with the experimental results of Qian. These outcomes were in good agreement with the experiments. The binary collisions of equal-sized droplets were investigated at various Weber numbers and impact parameters at room temperature and pressure conditions. The Weber number ranged from 0.5 to 200 and the impact parameters ranged from 0 to 1 , which covered three different types of outcomes: coalescence, head-on separation and off -centre separation. The results show that the critical Weber number of head-on collisions between coalescence and reflexive separation is 22 . The We-x schematic of various collision regimes of seawater droplets is obtained.
\end{abstract}

\section{INTRODUCTION}

ISSCT is widely used in the industrial and agricultural production due to small resistances, low maintenance costs and remarkable cooling effects, especially in thermal systems such as heat-engine plants. In the working process, the hot seawater is cooled by heat exchange with the circulatory in the tower, and then falls into the collecting basin [1]. The change of droplet size distribution will affect the performance of cooling tower. Therefore, the investigation of numerical simulation of droplet collision is providing a theoretical basis for the application of the ISSCT. The collision in the tower is a complicated multi-parameter two-phase flow problem. At present, lots of experiments and numerical simulation investigations of droplet collision have been carried out by domestic and oversea scholars (At present, some scholars have conducted a lot of experiments and numerical simulation studies on droplet collision). O'Rourke et al. [2] set up a statistical model of droplet collision based on the experimental results. Ashgriz and Poo [3] further improved the division of the collision outcomes region of droplet collision by experiments and it has been still used today. Numerical simulation has the advantage of capturing more details and analyzing the internal mechanism compared with experimentation. Nikolopoulos et al. [4-5] simulated the headon and off-centre collision of droplets by using adaptive mesh method and the result is similar to the experimental study by Qian and Law [6]. At present, most of the experimental and numerical investigations of droplet collision are both focused on hydrocarbon droplets [7-12], and there are still large gaps in concrete study of seawater droplets collision. In the present investigation, the feasibility and accuracy of numerical simulation are verified. Numerical simulation of head-on and off-centre binary collision between equal-sized seawater droplets is conducted using the Fluent. The mechanism and various collision regimes of seawater droplets are discussed, and the collision models of seawater droplets are presented.

\section{DROPLET COLLISION MECHANISM}

The phenomenon of binary droplet collision can be described based on liquid droplet density, viscosity, surface tension and other properties. By dimensional analysis, the above parameters can be grouped into four dimensionless numbers. They are Weber number (We), Reynolds number $(R e)$, non-dimensional impact parameter(x) and drop diameter ratio $(\triangle)$, which are defined as follows (1)-(4):

$$
\begin{aligned}
& W e=\frac{\rho u_{r}^{2} D_{0}}{\sigma} \\
& R e=\frac{\rho u_{r} D_{0}}{\mu} \\
& x=\frac{X}{D_{0}} \\
& \Delta=\frac{D_{S}}{D_{L}}
\end{aligned}
$$

where $\rho$ is droplet density; $D_{0}$ is droplet diameter; $u_{0}$ is droplet velocity; the relative droplet velocity $u_{r}=2 u_{0} ; \sigma$ is the surface tension coefficient of droplet; $\mu$ is viscosity; DS and DL respectively represent the diameter of small droplets and large droplets. In this paper, the droplet size is the same and drop diameter ratio is $1 . \mathrm{X}$ is defined as the distance from the centre of one droplet to the relative velocity vector placed on the 
centre of the other droplet, which is shown in Figure 1.

In a more detailed experiment, Qian and Law [6] used a series of time-resolved photo images to map all collision states to determine the factors of different results. Five distinct regimes of collision outcomes were concluded, namely I) coalescence after minor deformation; II) bouncing; III) coalescence after substantial deformation; IV) Reflexive separation; V) Stretching separation.

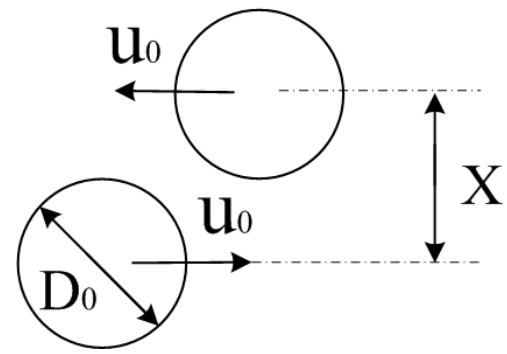

Figure 1. Schematic of the droplet collision

The schematic of collision regimes with various Weber numbers and impact parameters is presented. The gas between droplets must be compressed to such an extent that the droplets gap is reduced to a dimension comparable to that of the intermolecular force in order for the droplets to coalesce. When two droplets are approaching each other at a very slow rate, the pressure between the two drops increases. The gas hasn't expelled before the droplets come into contact, and the droplets will coalesce under Van der Waals force. When the two drops come very close, the pressure between the gases is going to increase, these gases will form a gas film to stop the droplets from moving closer together. If the two droplets are not in contact until the relative rate is zero, the two droplets bounce off each other. When the rate of droplet continues to increase, the gas film between droplets is not enough to prevent the droplets from contacting directly. Because of viscous dissipation and surface tension, the droplet rate decreases, and the internal flow does not have enough kinetic energy to separate the two drops, causing the droplets to bond. With the larger of Weber number, the combined droplets still have enough kinetic energy to break the combined large droplets, leading to separation and the simultaneous formation of a tiny satellite droplet. According the relative position of two droplets contact, the separation is divided into reflexive separation and stretching separation.

\section{CONTROL EQUATIONS AND COMPUTATIONAL MODELS}

The crux to the numerical simulation of droplets collision is to capture gas-liquid interface. The numerical simulation method is divided into grid method and particle method. VOF method [13-14] formulates interface by defining the volume fraction of each phase in the grid. It is a surface tracking method under a fixed Euler grid, which can be used to solve steady or unsteady interface. Fluids are simulated by solving momentum equations and by tracking the volume fraction of each fluid through the calculation domain. It has the advantages of good conservation and high simulation accuracy. Therefore, VOF method is used to track the gasliquid interface of droplet collision in this paper. The fluid volume function $\alpha$ is defined as the volume fraction of the first phase accounted in the computational grid.

$$
\alpha\left\{\begin{array}{l}
1, \text { inside liquid phase } \\
0, \text { inside gas phase } \\
0<\alpha<1 \text {, inside the transitional area between the two phase }
\end{array}\right.
$$

The value of density $\rho$ and viscosity $\mu$ are calculated using linear interpolation between the values of the two phases weighted with the volume fraction $\alpha$ :

$$
\begin{aligned}
& \rho(\alpha)=\alpha \rho_{l}+(1-\alpha) \rho_{g} \\
& \mu(\alpha)=\alpha \mu_{l}+(1-\alpha) \mu_{g}
\end{aligned}
$$

where the subscript 1 and $g$ represent the liquid phase and the gas phase, respectively. The transport equation for the volume function is

$\frac{\partial \alpha}{\partial t}+\nabla \cdot(\boldsymbol{u} \alpha)=0$

It is necessary to consider the liquid viscous and surface tension of the seawater droplets collision in ISSCT, and the flow is incompressible flow. Energy equation is not solved since heat transfer is not considered. Continuous equation is as follows:

$$
\nabla \cdot \boldsymbol{u}=0
$$

The momentum equation expressing both phases is written in the form:

$\rho\left(\frac{\partial \boldsymbol{u}}{\partial t}+\boldsymbol{u} \cdot \nabla \boldsymbol{u}\right)=-\nabla p+\nabla \cdot(2 \mu \boldsymbol{D})+F_{S}$

where $u$ is the velocity vector; $p$ is the pressure; $\mu$ is dynamic viscosity; $\rho$ is the density; $D$ is the stress tensor. The expression is $\mathrm{Dij}=(\partial \mathrm{ui} / \partial \mathrm{xj}+\partial \mathrm{uj} / \partial \mathrm{xi}) / 2$ and $\mathrm{Fs}$ is the volume force caused by surface tension. The continuous surface tension CSF model proposed by Brackbill [15] is chosen and the expression is $F \mathbf{s}=\sigma \kappa n$, where $\sigma$ is coefficient of surface tension; $\kappa$ is the curvature of the gas-liquid interface; $n$ is interface unit normal vector. Three-dimensional problem can be simplified as a two-dimensional problem due to the symmetry of the calculation model, and the axisymmetric model can be used to calculate. The adaptive mesh method is used to encrypt the region with obvious gradient change at the interface of two phases (Due to the symmetry of the calculation model, the three-dimensional problem can be simplified into a two-dimensional one. The axisymmetric model is used for calculation, and the region with obvious gradient change of the two-phase interface is encrypted with the adaptive grid method). The computing domain increases with the droplet velocity. The size range is $3 \mathrm{D} 0 \times 1.5 \mathrm{D} 0$ to 12 D0 66 D0, and the distance between droplets is 0.5 D0. The bottom boundary is the axisymmetric boundary and the other boundaries are wall boundary. The PISO pressure-velocity coupling algorithm and PRESTO pressure interpolation format are simulated. 


\section{NUMERICAL METHOD VALIDATION}

In order to investigate the grid dependency of the results, the droplets collision with minimum grid size of D0/200, D0/300 and D0/400 is simulated. The numerical results indicate that the hydrodynamic behavior of the impacting droplets does not change when the minimum grid size is D0/200. And then the tetradecane droplet collisions experiment conducted by Qian and Law is simulated, and the calculation conditions are in accordance with the experimental conditions, $\mathrm{We}=32.8, \mathrm{Re}=210.8, \mathrm{D} 0=318 \mu \mathrm{m}$.

Figure 2 shows the comparison between the numerical simulation and experiment, the deformations of the droplet are basically agreed. Therefore, VOF model can be used to simulate the collision process of binary droplet.

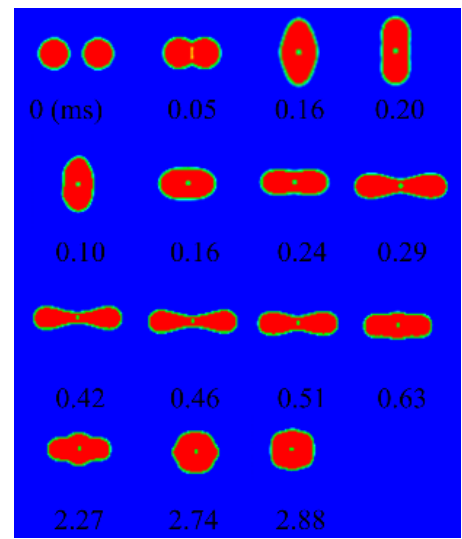

(a) Simulation results

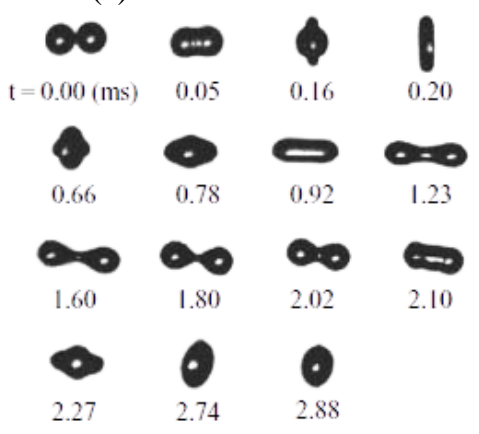

(b) Experimental results

Figure 2. Comparison between numerical simulation and experimental results from Qian

\section{RESULTS AND DISCUSSES}

\subsection{Physical properties of seawater droplets}

Seawater is a mixture of dissolved inorganic salts, organics, gases and a large amount of suspended substances. The concentration of seawater is about 35\%. Seawater droplet diameter is generally between hundreds of microns and hundreds of millimeters in ISSCT. In the present investigation, seawater droplets with a diameter of $200 \mu \mathrm{m}$ are chosen for the computation. Table 1 shows the properties of seawater droplet and medium gas, which are measured at ambient temperature and pressure. Empirical relations refer to [16-19].

The head-on and off-centre binary collisions of equal-sized droplets are simulated at various Weber numbers. The Weber number is transformed by changing the relative droplet velocity. The initial velocities of the two droplets are the same but in opposite directions. The parameters of the droplet collision are $\mathrm{We}=0.5 \sim 100$, $\mathrm{ur}=0.42 \sim 8.44 \mathrm{~m} / \mathrm{s}, \mathrm{D} 0=200 \mu \mathrm{m}$.

Table 1. Physical properties of seawater droplets and air

\begin{tabular}{cccc}
\hline & $\begin{array}{c}\text { Density } \\
\left(\mathrm{kg} / \mathrm{m}^{3}\right)\end{array}$ & $\begin{array}{c}\text { Viscosity } \\
(\mathrm{Pa} \cdot \mathrm{s})\end{array}$ & Surface tension $(\mathrm{N} / \mathrm{m})$ \\
\hline seawater & 1021.53 & $0.963 \times 10^{-3}$ & 0.07281 \\
air & 1.225 & $1.834 \times 10^{-5}$ & \\
\hline
\end{tabular}

\subsection{Head-on collision of seawater droplets}

Figure 3 shows the head-on collision of the seawater droplets at various Weber numbers.

The head-on collision of the seawater droplets is shown in Figure 3. In Figure 3(a), when two droplets are approaching each other at a very slow rate, the kinetic energy of the droplet is larger than gas pressure in the gap. Two droplets begin to coalesce at the edge of the contact, forming a stretched cylinder. The gas between two droplets trapped by the droplets is absorbed into the large droplet, forming a small bubble, as shown at $0.20 \mathrm{~ms}$ in Figure 3(a). At $0.36 \mathrm{~ms}$, the surface energy reaches the maximum while the kinetic is in its minimum. Then, the liquid drop change from radial motion to axial motion, and the kinetic energy increase. However, the kinetic energy of the internal flow is insufficient to overcome the surface tension, and the fracture is connected to the neck. After several axial and radial vibrations, a stable large liquid drop is formed. It is shown in Figure 3(b), as Weber number increases, the coalescence time of droplets is significantly advanced. The larger kinetic energy leads to greater droplet deformation, and the oscillation period of the coalesced droplet becomes longer until a spherical droplet is formed. The onset of reflexive separation is at $\mathrm{We}=22$, as shown in Figure 3(c). The bubble is squeezed out due to the extreme deformation in radial direction.

Figure 3(d) shows a typical reflexive separation result at $\mathrm{We}=23$. The deformation before droplet separation is very similar to the deformation process of Figure 3(c), but the radial deformation is much greater. The droplet internal fluid converges to the edge and the center is stretched thin. Then, the droplet motions in axial. The droplet has enough kinetic energy to overcome surface tension force, leading to separation. With the increase of Weber number, the two ends of the droplets separate and the simultaneous formation of a tiny satellite droplet is shown in Figure 3(e). This pinching action can also be seen clearly in Figure 3(f), in which a larger satellite droplet is formed as the connecting neck is pinched off. The satellite droplet is roughly in the same diameter as its two ends (This compression is also clearly seen in figure 3(f), where large satellite droplets are formed as a result of the extrusion of the neck and the diameter of the satellite drops is approximately the same as that at both ends).

With further increasing Weber number, the droplet separation time is gradually shortened, and the number of satellite droplets is increasing. There are three, five and seven droplets formed respectively in Figure 3(g)-(i). The two ends of the droplet gradually break off and then the newly formed ends are pinched off, forming new droplets. The mechanism of separation is the end-pinching mechanism, rather than the capillary instability mechanism, which agrees well with the results of literature [19]. 


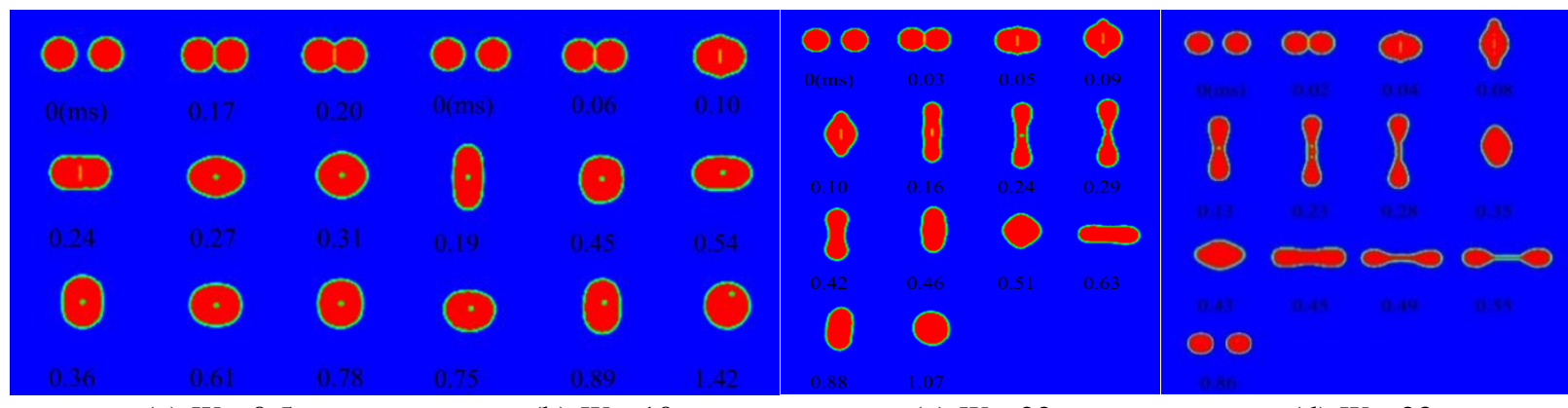

(a) $W e=0.5$

(b) $W e=10$

(c) $W e=22$

(d) $W e=23$

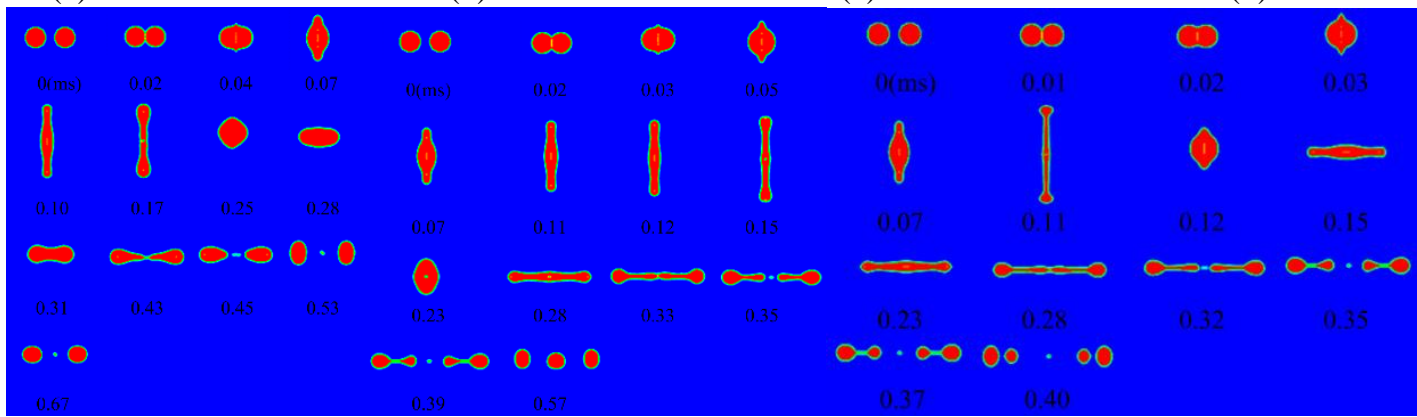

(e) $W e=50$

(f) $W e=80$

(g) $W e=100$

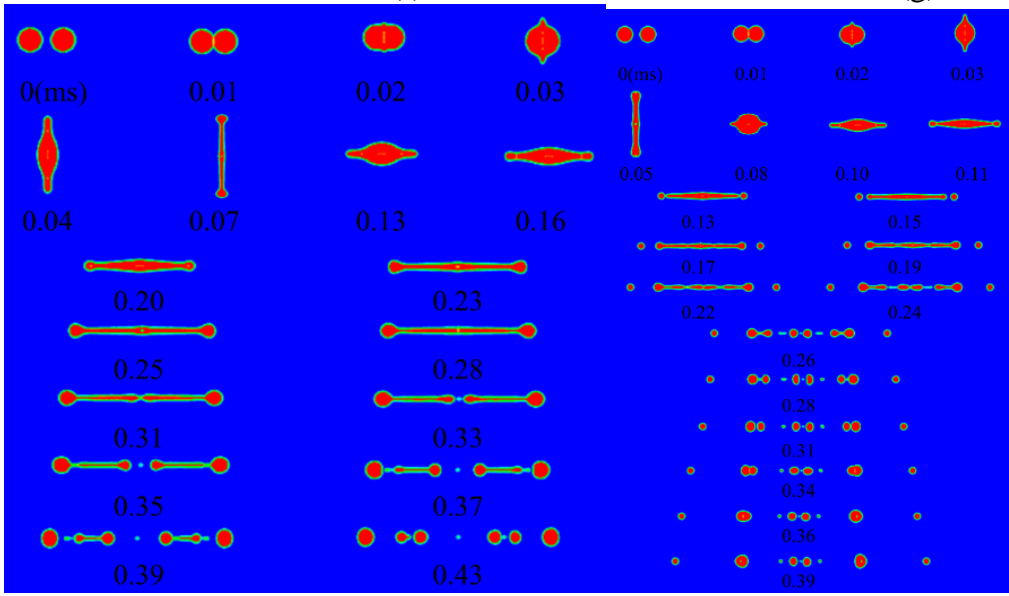

(h) $W e=150$

(i) $W e=200$

Figure 3. Numerical simulation of head-on collisions of seawater droplets at various Weber numbers

\subsection{Off-centre collision of seawater droplets}

In order to summarize the We-x schematic of various collision regimes of seawater droplets, the numerical simulation of the droplets off-centre collision is conducted at various $\mathrm{x}$. The collision results at $\mathrm{We}=50$ are shown in Figure 4.

Figure 4(a) shows the reflexive separation case at $x=0.1$. The droplet reaches the maximum radial deformation in $0.12 \mathrm{~ms}$ and then begins to contract in radial direction. The droplet is separated at $0.45 \mathrm{~ms}$. The gap between the two drops is longer than the gap of head-on collision in the same We due to the rotation motion (Because of the rotational motion, the gap between the two droplets is longer than that of an oncoming collision). By comparing $0.13 \mathrm{~ms}$ and $0.23 \mathrm{~ms}$ in Figure 4(b), it can be seen clearly that the droplets rotation angle increases with the larger $\mathrm{x}$. The collision outcomes shown in Figure 4(c) and (d) are stretching separation. With the increase of $\mathrm{x}$, the rotation period of droplets becomes significantly longer. The connecting neck is getting thinner and thinner and breaking into satellite droplets under the force of centrifugation and inertia, at $\mathrm{x}=0.3$.

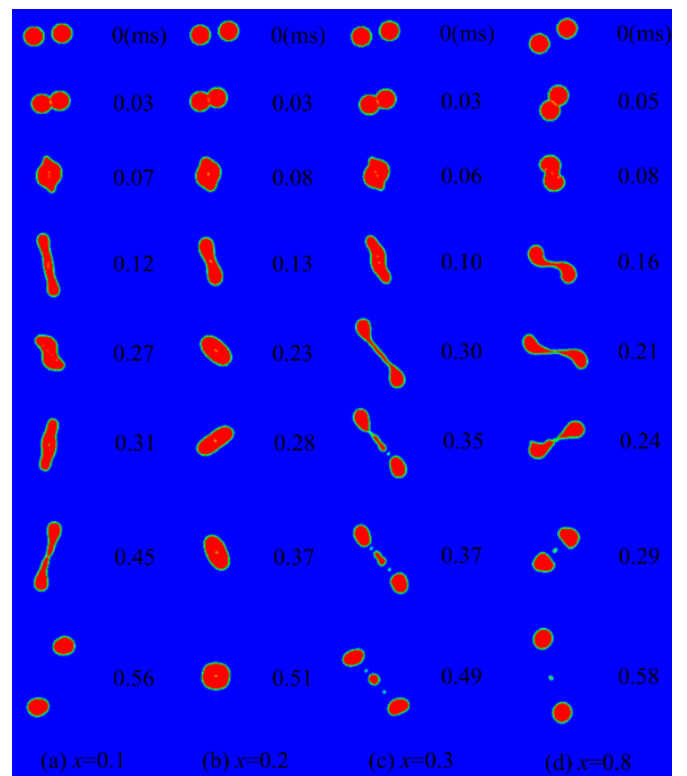

Figure 4. Numerical simulation of seawater droplets collision at various impact parameters 
In Figure 4(d), the non-overlapping droplets at both ends continue to move in the opposite direction, and the centrifugal force is more important than the inertial force. Due to the high influence parameters, the deformation of droplets is small and the rotation amplitude increases significantly. The connecting neck is broken and two equal-size parent droplets are formed [20-21].

\subsection{Regime schematic of numerical results for collision of seawater droplets}

According to the numerical simulation of the seawater droplets collision, the We ranges from 0.5 to 200 and $\mathrm{x}$ ranges from 0 to 1 . The schematic of various collision regimes of equal-sized seawater droplets is obtained, as shown in Figure 5 , and three different outcomes are obtained, namely coalescence, reflexive separation and stretching separation (Figure 5 shows the schematic diagram of different collision mechanisms of seawater droplet at the same scale, and three different results are obtained: polymerization, self-reverse separation and tensile separation).

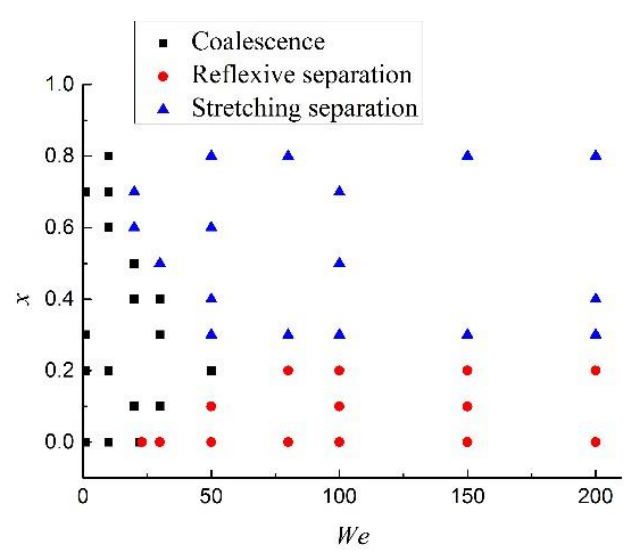

Figure 5. Schematic of various collision regimes of seawater droplets

\section{CONCLUSION}

In the present paper, detailed comparison with timeresolved image from Qian and Law is used to validate the numerical model. And then, numerical simulations of binary collision between equal-sized seawater droplets are conducted at various Weber numbers and impact parameters. The We-X schematic of various collision outcomes of seawater droplets is obtained. The conclusions are as follows:

(1) Numerical simulation of the head-on collision of tetradecane droplets in the nitrogen environment is conducted. Validate VOF method can be used to track the interface.

(2) Numerical simulation of the head-on collision of seawater droplets is conducted at various Weber numbers. The critical Weber number of head-on collisions between coalescence and reflexive separation is 22 .

(3) Reflexive separation at Weber number is less than 200. As Weber number increases, the number of satellite droplets increases, and the mechanism of reflexive separation is called the end-pinching mechanism.

(4) The seawater droplets coalescence and reflexive separation mechanism between head-on collision and off- centre collision are the same, but the critical Weber number between coalescence and reflexive increases due to rotation motion.

\section{ACKNOWLEDGMENT}

The financial support of the Nation Natural Science Foundation of China (grant no. 51879154 and 51806128) is acknowledged.

\section{REFERENCES}

[1] Wei Z, Qi X. (2013). Investigation on equivalent diameter of water droplets in showing cooling tower. Science Technology and Engineering 13(17): 47974800 .

[2] O'Rourke PJ. (1989). Statistical properties and numerical implementation of a model for droplet dispersion in a turbulent gas. Journal of Computational Physics 83(2): 345-360. https://doi.org/10.1016/0021-9991(89)90123$\mathrm{X}$

[3] Ashgriz N, Poo JY. (2006). Coalescence and separation in binary collisions of liquid drop. Journal of Fluid Mechanics 221: 183-204. https://doi.org/10.1017/S0022112090003536

[4] Nikolopoulos N, Nikas KS, Bergeles G. (2009). A numerical investigation of central binary collision of droplets. Computers \& Fluids 38(6): 1191-1202. https://doi.org/10.1016/j.compfluid.2008.11.007

[5] Nikolopoulos N, Theodorakakos A, Bergeles G. (2009). Off-centre binary collision of droplets: A numerical investigation. International Journal of Heat and Mass Transfer 52(19-20): 4160-4174. https://doi.org/10.1016/j.ijheatmasstransfer.2009.04.011

[6] Qian J, Law CK. (1997). Regimes of coalescence and separation in droplet collision. Journal of Fluid Mechanics 331(01): 59-80. https://doi.org/10.1017/S0022112096003722

[7] Brazier-Smith PR, Jennings SG, Latham J. (1972). The interaction of falling water drops: coalescence. Proceedings of the Royal Society of London 326(1566): 393-408. https://doi.org/10.1098/rspa.1972.0016

[8] Jiang YJ, Umemura A, Law CK. (1992). An experiment investigation on the collision behavior of hydrocarbon droplets. Journal of Fluid Mechanics 234: 171-190.

[9] Pan Y, Suga K. (2005). Numerical simulation of binary liquid droplet collision. Physics of Fluids 17(8): 082105.

[10] Qiang HF, Shi C, Chen FZ, Han YW. (2013). Simulation of two-dimensional droplet collisions based on $\mathrm{SPH}$ method of multi-phase flows with large density differences. Acta Phys. Sin. 62(21): 245-259. https://doi.org/10.7498/aps.62.214701

[11] Liu ZJ, Zhang C, Wu LL, Wu JJ. (2015). Numerical simulation of head-on binary collision of droplets in gas phase environment. Chinese Journal of Computational Mechanics 32(1): 136-141.

[12] Yin J, Kong W, Wang FX, Liu H, Yang K. (2017). Experimental investigation of binary supercooled water droplet collision. Journal of Shanghai Jiao Tong University 51(8): 939-945. 
[13] Hirt CW, Nichols BD. (1981). Volume of fluid (VOF) method for the dynamics of free boundaries. Journal of Computational Physics 39(1): 201-225.

[14] Wang J, Li QH, Lu M. (2003). Numerical method for free-surface fluid flow-a review. Chinese Journal of Computational Mechanics 2(1): 101-108.

[15] Brackbill JU, Kothe DB, Zemach C. (1992). A continuum method for modeling surface tension. Academic Press Professional, Inc. 100(2): 335-354. https://doi.org/10.1016/0021-9991(92)90240-Y

[16] Zheng X. (2018). Numerical simulation of aseismatic reinforced concrete frame structure with fiber reinforced plastics. Chemical Engineering Transactions 66: 11411146. https://doi.org/10.3303/CET1866191
[17] Isdale JD, Morris R. (1972). Physical properties of sea water solutions: density. Desalination 10(4): 329-339.

[18] Isdale JD, Spence CM, Tudhope JS. (1972). Physical properties of sea water solutions: viscosity. Desalination 10(4): 319-328.

[19] Qi XN. (2008) Research on the shower cooling tower. Shanghai Jiao Tong University.

[20] Chen S. (2018). Numerical simulation of split-hopkinson pressure bar test on high-density polyethylene. Chemical Engineering Transactions 66: 271-276 https://doi.org/10.3303/CET1866046

[21] Xia SY, Hu CB. (2014). Direct numerical simulation of head-on binary collision of aluminum oxide droplet. Applied Mathematics and Mechanics 35(04): 377-388. 